\title{
FIB Applications: A Historical Perspective
}

\author{
F. A. Stevie ${ }^{1}$ and L. A. Giannuzzi ${ }^{2}$ \\ ${ }^{1}$ Analytical Instrumentation Facility, North Carolina State University, Raleigh, NC 27695, USA \\ ${ }^{2}$ EXpressLO LLC, Fort Myers, Florida 33913, USA
}

FIB applications were initially driven by the silicon semiconductor industry and concentrated on preparation for SEM and modification of devices and masks. Development of TEM preparation coupled with the site specific capability provided by FIB significantly enhanced failure analysis for $\mathrm{Si}$ technology and in lightwave materials such as InP [1]. Initial TEM preparation still required mechanical polishing [2], but the use of a micromanipulator to lift-out a specimen was successful on the first attempt [3]. FIG. 1 illustrates steps in this ex situ procedure to obtain a specimen from a device that had wire bonding issues and place the specimen on a carbon film TEM grid. The specimen had been cut free from the substrate to permit the lift-out.

The development of a routine method for lift-out sample preparation, coupled with a local concentration of instruments and analysts, fostered an explosion of applications [4-6]. Lift-out specimens were prepared for TEM, SIMS, AES, and XPS analyses [5]. An improved understanding was achieved for sputtering, contrast mechanisms and protection of the surface [7]. Methods to further reduce sample preparation time were developed and automatic operation of instruments led to multiple sample preparation overnight. Analysis expanded into a wide range of materials, from semiconductors to optical materials, from plastics to particles [8]. in situ lift out can also be done with an internal micromanipulator as shown in FIG. 2 [8]. Dual platform FIB-SEM instruments provided 3D analysis and improvement in the ability to prepare the thinnest possible TEM specimens [9]. ex situ lift out is also appropriate for usage with MEMS carrier devices as shown in FIG. 3a. One disadvantage of the conventional ex situ lift-out method was that the sample could not be thinned further after removal. Recent advances in specimen grid designs and techniques also allow further thinning with ex situ specimen removal as shown in FIG. 3b [10-12]. The FIB $\mathrm{Ga}^{+}$ion beam size may have reached a practical limit but the introduction of plasma ion sources and TOF-SIMS detector show that advances in this field are far from complete $[13,14]$. The high current capability of the plasma sources permits 100 $\mu \mathrm{m}$ deep cuts that improve the analysis of coatings [15]. Other developments continue such as new gases for enhanced etch or specific applications. Smaller diameter plasma sources are on the horizon and cryogenic analysis for biological specimens is in its infancy.

[1] R. Hull et al., Appl. Phys. Lett. 62 (1993) p. 3408.

[2] F. A. Stevie et al., Surf. Int. Anal. 23 (1995) p. 61.

[3] L. A. Giannuzzi et al., Mat. Res. Soc. Symp. Proc., 480 (1997) p. 19.

[4] L. A. Giannuzzi and F. A. Stevie, Micron 30 (1999) p. 197.

[5] F. A. Stevie et al., Microsc. Microanal. 5, (S2) (1999) p. 888.

[6] F. A. Stevie et al., Surf. Int. Anal. 31 (2001) p. 345-351.

[7] B. W. Kempshall et al., J. Vac. Sci. Technol. B20 (2002) p. 286.

[8] "Introduction to Focused Ion Beams: Theory, Instrumentation, Applications, and Practice",L.A.

Giannuzzi and F.A Stevie, eds., Springer, NY (2005).

[9] L.A. Giannuzzi et al., Microsc Microanal 9(S 2) (2005) p. 828.

[10] L.A. Giannuzzi, Microsc. Microanal. 18 (S2) (2012) p. 632. 
[11] L.A. Giannuzzi, Proc. ISTFA (2012) p. 388.

[12] L.A. Giannuzzi, Microsc. Microanal. 19 (S2) (2013) p. 906.

[13] J. A. Whitby et al., Advances in Materials Science and Engineering, 2012 (2012) Article ID 180437.

[14] F. A. Stevie et al., (in press).Surf. Int. Anal. (2014).

[15] L.A. Giannuzzi et al., Microsc. Microanal. 19 (S2) (2013) p. 1862.
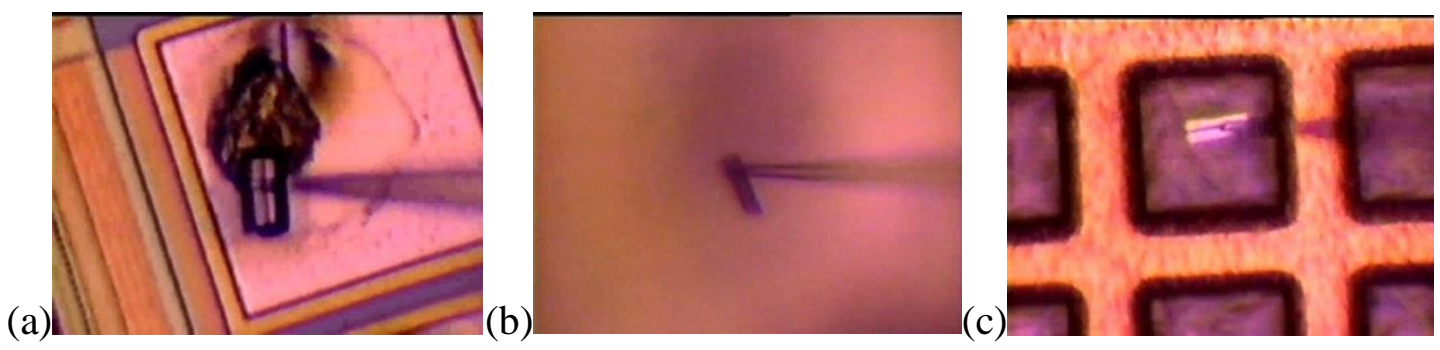

Figure 1. ex situ lift out showing (a) manipulator acquisition of specimen, (b) specimen on manipulator needle, and (c) transfer of specimen to a carbon coated TEM grid.

(a)

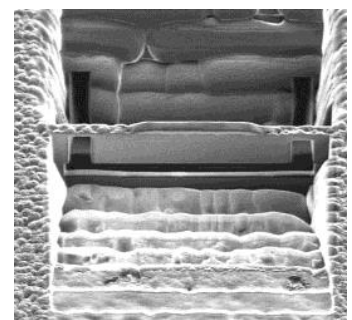

(b)

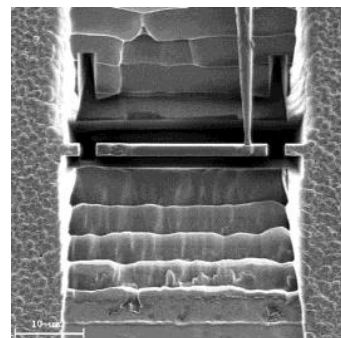

(c)

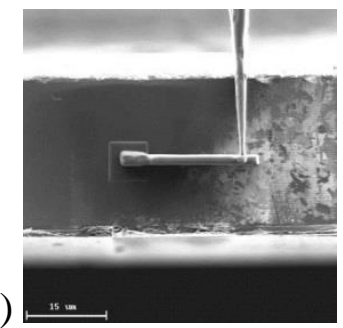

(d)

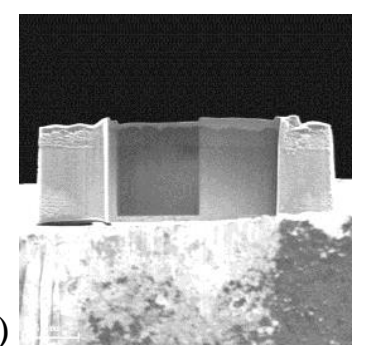

Figure 2. Microsampling from an Hitachi FIB or in situ lift out showing (a) cuts, (b) needle attachment and specimen release, (c) specimen on grid, (d) specimen thinned to two different thichnesses. Dale Batchelor, Analytical Instrumentation Facility, North Carolina State University

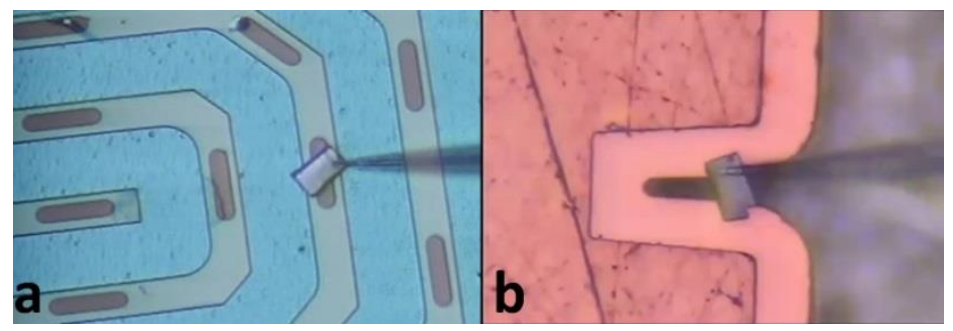

Figure 3. (a) ex situ lift out showing manipulation to a MEMS device carrier (materials courtesy of Qiang Xu, DENSsolutions). (b) ex situ lift out onto a new grid design [10-12]. 